\title{
Dynamical analysis of seemingly interaction-free measurements
}

\author{
A. Luis and L. L. Sánchez-Soto \\ Departamento de Óptica, Facultad de Ciencias Físicas, Universidad Complutense, 28040 Madrid, Spain
}

(Received 16 December 1997)

\begin{abstract}
A full quantum analysis of $100 \%$ efficient interaction-free measurements is presented. It is shown that it is possible to describe consistently the phenomenon in terms of an effective interaction between object and apparatus that disturbs measurable quantities of the observed system. [S1050-2947(98)08007-X]

PACS number(s): $03.65 . \mathrm{Bz}, 42.50 . \mathrm{Dv}$
\end{abstract}

\section{INTRODUCTION}

It is customary to think that any measurement always involves an interaction that changes the state of the apparatus according to the actual state of the observed system. This applies to both quantum and classical detections, although, unlike classical physics, quantum theory seems also to impose that the observed system is unavoidably disturbed by the measurement.

However, it has been recently discovered that it is possible to ascertain the existence of an object in a given region of the space seemingly without interacting with it $[1,2]$. This remarkable phenomenon seems to defy basic convictions. For instance, if there is no interaction it can be asked whether the observed system is disturbed or not. If it is disturbed, the question is how this can occur without an effective interaction [3]. The aim of the present work is to examine whether these interaction-free measurements actually contradict or not the previous beliefs concerning quantum detection.

A well-studied realization employs a Mach-Zehnder interferometer that can contain the object to be detected in one of its arms [1]. The interferometer is arranged in such a way that, in the absence of the object, the photon (or any other interfering particle) will always exit via a given output port (bright port) and never via the other one (dark port). The presence of the object, assumed to be a perfect absorber or scatterer, destroys the interference so that there is some probability that the photon will exit via the dark output port. When this occurs, it can be concluded that the object was certainly there and also that the photon has not impinged on the object: had the photon struck the object it would have been removed from the interferometer. To make the argument more dramatic the object is sometimes pictured as a light-sensitive bomb that would explode if struck by one photon.

Although a quantum detection without interaction might be regarded as paradoxical, it could be argued that this is an effect of fundamental quantum peculiarities. In fact, this kind of detection is impossible classically, since it relies on a suitable balance between wave and corpuscular behaviors of the interfering particle [2].

It can be noticed that, while it is not possible to explain the whole phenomenon by using either a corpuscular or a wave picture, the absence of interaction relies solely on a corpuscular description [4]. This remark leaves room for the existence of an actual effective interaction. Although it will not involve the actual exchange of energy quanta between apparatus and object, some other possibilities might still be open, so that the effect of an actual interaction can be the change of any other dynamical variables.

A trace of an object-apparatus interaction is the change of some measurable property of the object. To disclose it, the object should be treated quantum mechanically, as opposed to the classical descriptions considered in previous works. A fully quantum treatment allows one to include the possibility of a coherent superposition of the object-in and object-out situations. This is relevant because it can be expected that the detection process will lead to the replacement of such superposition by the corresponding statistical mixture. From a dynamical perspective, this decoherence should be the effect of an object-apparatus interaction disturbing the relative phase of the quantum superposition. Moreover, it is known that a classical description of part of a detection arrangement can lead to wrong conclusions [5].

Our purpose is to carry out a full quantum analysis of the evolution of the object-apparatus system that should reveal these dynamical features of the process. We will focus on $100 \%$ efficient interaction-free detections $[2,6]$. In this way we can avoid dealing with single events, which can be regarded as statistical outcomes of a process that is not interaction free when considering ensemble averages after a large number of trials $[1,6]$. On the other hand, single events explicitly involve the actual realization of a measurement on the apparatus, whose nonlocality and nonunitary collapse might overwhelm the dynamical details we are looking for.

In Sec. II we present an all-optical realization of a $100 \%$ interaction-free measurement. The object to be detected is a photon in a given electromagnetic field mode. This example allows one to consider naturally the coherent superposition of the object-in and object-out situations. In Sec. III we analyze the effective interaction accounting for the changes experienced by the object and the apparatus during the detection process. The change experienced by the object state is a measurable effect, as it is shown in Sec. IV.

\section{A SEEMINGLY INTERACTION-FREE MEASUREMENT WITH QUANTUM OBJECT}

The purpose of the detection is to ascertain the presence or absence of a photon in a given electromagnetic field mode $b$. The one-photon state $|1\rangle_{b}$ and the vacuum $|0\rangle_{b}$ represent the object-in and object-out situations, respectively. Their coherent superposition $\alpha|0\rangle_{b}+\beta|1\rangle_{b}$, with $|\alpha|^{2}+|\beta|^{2}=1$, is the most general pure state for the object. 


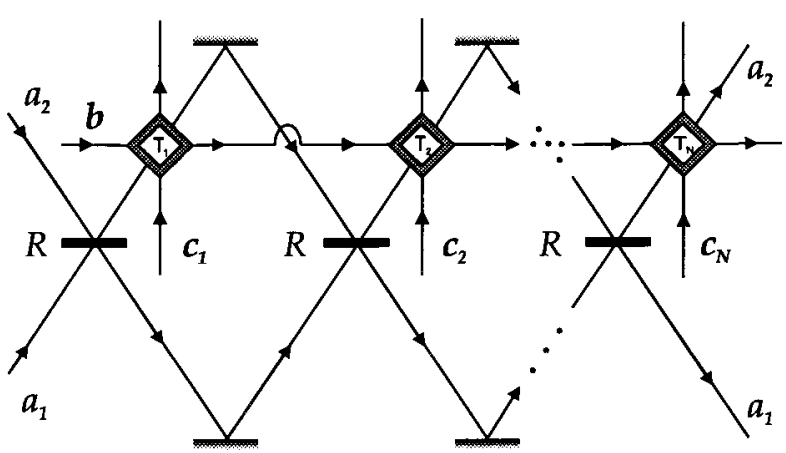

FIG. 1. Schematic of an interaction-free detection by repeated interrogation devised to detect the presence or absence of a photon in mode $b$. A single photon in mode $a_{1}$ incides on a series of $N$ connected Mach-Zehnder interferometers. After each beam splitter $R$ the upper path (mode $a_{2}$ ) is coupled by a Kerr interaction in $T_{j}$ with mode $b$ and auxiliary modes $c_{j}$ in vacuum. If there is no photon in $b$ nothing happens in any $T_{j}$. If there is a photon in $b$, the photon in mode $a_{2}$ impinging on $T_{j}$ will be transferred to a mode $c_{j}$ being removed from the interferometer.

As in the previously considered realizations, the apparatus consists of a two-mode interferometer illuminated by a single interfering photon. A $100 \%$ detection efficiency can be approached using the method of repeated interrogation [2] illustrated in Fig. 1. A single photon in mode $a_{1}$ is incident from an input port of a series of $N$ Mach-Zehnder interferometers connected by identical beam splitters $R$ of reflectivity $\cos ^{2} \epsilon$. The relative phases between paths in the upper and lower halves are zero. Denoting by $a_{1}$ and $a_{2}$ the complex amplitude operators corresponding to the lower and upper paths, respectively, their coupling at each beam splitter is described by the unitary operator

$$
R=e^{\epsilon\left(a_{2}^{\dagger} a_{1}-a_{1}^{\dagger} a_{2}\right)}
$$

After each beam splitter the mode $a_{2}$ is coupled in $T_{j}$ with mode $b$ and auxiliary field modes $c_{j}(j=1, \ldots, N)$ initially in vacuum. This coupling must discriminate between the presence or absence of the photon in mode $b$. This can be done by considering a nonlinear Kerr coupling described by the unitary operator

$$
T_{j}=e^{(\pi / 2) b^{\dagger} b\left(c_{j}^{\dagger} a_{2}-a_{2}^{\dagger} c_{j}\right)}
$$

which can be regarded as a beam splitter for modes $a_{2}$ and $c_{j}$ controlled by the photon number in mode $b$. If there is no photon in $b$ (object out) nothing happens in any $T_{j}$ :

$$
T_{j}|0\rangle_{b}|1\rangle_{a_{2}}|0\rangle_{c_{j}}=|0\rangle_{b}|1\rangle_{a_{2}}|0\rangle_{c_{j}}
$$

the photon remains in mode $a_{2}$ and the interference continues without disturbances. When there is a photon in $b$ (object in), the photon in mode $a_{2}$ is transferred to the mode $c_{j}$ :

$$
T_{j}|1\rangle_{b}|1\rangle_{a_{2}}|0\rangle_{c_{j}}=|1\rangle_{b}|0\rangle_{a_{2}}|1\rangle_{c_{j}}
$$

and is removed from the interferometer. Therefore, the combination of the photon in $b$ with the Kerr medium acts as a perfect absorber or scatterer of the interfering photon. More dramatically, the Kerr medium might be regarded as a bomb loaded by the $b$ photon and ready to be triggered by a photon in mode $a_{2}$ impinging on it. The appearance of a photon in modes $c_{j}$ represents its explosion. Then, the simultaneous coincidence of the two photons in the nonlinear medium is the object-apparatus interaction that an interaction-free detection attempts to avoid.

Let us show how the whole arrangement works by calculating the output field state. The initial state is assumed to be

$$
|\Psi\rangle=\left(\alpha|0\rangle_{b}+\beta|1\rangle_{b}\right)|1,0\rangle_{a}|0, \ldots, 0\rangle_{c},
$$

where $|n, m\rangle_{a}=|n\rangle_{a_{1}}|m\rangle_{a_{2}}$. The output state is the result of the action of the unitary operator $U=T_{N} R T_{N-1} R \cdots T_{1} R$ on the input state $|\Psi\rangle$,

$$
\begin{aligned}
U|\Psi\rangle= & \alpha|0\rangle_{b}\left[\cos (N \epsilon)|1,0\rangle_{a}+\sin (N \epsilon)|0,1\rangle_{a}\right]|0, \ldots, 0\rangle_{c} \\
& +\beta[\cos (\epsilon)]^{N}|1\rangle_{b}|1,0\rangle_{a}|0, \ldots, 0\rangle_{c}+\beta \sin (\epsilon) \\
& \times|1\rangle_{b}|0,0\rangle_{a} \sum_{j=1}^{N}[\cos (\epsilon)]^{j-1}\left|0, \ldots, 1_{j}, \ldots, 0\right\rangle_{c} .
\end{aligned}
$$

The first term represents the unobstructed interferometer: if $\epsilon=\pi /(2 N)$ the interfering photon will appear always in the bright output port in mode $a_{2}$. The other two terms correspond to the presence of the object. The interfering photon appears in the dark output in mode $a_{1}$ (interaction-free detection) or is removed from the interferometer (interaction).

The coincidence of the object and the interfering photon occurs with a probability

$$
P=|\beta|^{2}\left\{1-\left[\cos \left(\frac{\pi}{2 N}\right)\right]^{2 N}\right\} .
$$

In order to have a $100 \%$ efficient interaction-free measurement $P$ should approach zero. This occurs in the limit of large $N$ since when $N \rightarrow \infty$ we have $P \simeq|\beta|^{2} \pi^{2} /(4 N) \rightarrow 0$ and

$$
U|\Psi\rangle \rightarrow\left(\alpha|0\rangle_{b}|0,1\rangle_{a}+\beta|1\rangle_{b}|1,0\rangle_{a}\right)|0, \ldots, 0\rangle_{c}
$$

In this limit the modes $c_{j}$ will remain always in vacuum. The appearance of the interfering photon at the dark or bright port reveals the presence or absence of the object respectively.

\section{EFFECTIVE INTERACTION}

The final state (2.8) shows the usual entanglement correlating object and apparatus. This implies that object and apparatus observables have changed. Although the state (2.8) contains the relevant information concerning the detection, no actual measurement has been carried out. This means that the changes experienced by the object and the apparatus are amenable to a purely dynamical explanation.

Interaction-free measurement occurs provided that modes $a_{2}$ and $c_{j}$ are initially in vacuum. We can take this into account explicitly and simplify the expression for $U$ in the limit $N \rightarrow \infty$ in the form 


$$
U|\varphi\rangle_{b}|\psi, 0\rangle_{a}|0, \ldots, 0\rangle_{c} \rightarrow|0, \ldots, 0\rangle_{c} \tilde{U}|\varphi\rangle_{b}|\psi, 0\rangle_{a}
$$

where $|\psi\rangle$ and $|\varphi\rangle$ are arbitrary field states in the corresponding modes. Taking $\epsilon=\delta / N$, with $\delta$ constant, we have

$$
\widetilde{U}=e^{(\delta / 4)\left\{1+\cos \left(\pi b^{\dagger} b\right)+2 \cos \left[(\pi / 2) b^{\dagger} b\right]\right\}\left(a_{2}^{\dagger} a_{1}-a_{1}^{\dagger} a_{2}\right)} .
$$

The field state after $N / k$ beam splitters can be also obtained from Eqs. (3.1) and (3.2) by replacing $\delta$ by $\delta / k$. For any $\delta$, $\widetilde{U}$ is a unitary transformation involving just modes $a_{1}, a_{2}$, and $b$. This provides a simple dynamical description of the phenomenon in terms of an effective interaction responsible for the existence of observation and the changes suffered by object and apparatus measurable quantities. It can be seen that $\widetilde{U}$ represents a standard SU(2) interferometer controlled by the photon number $b^{\dagger} b$ [7]. It commutes with the object and apparatus photon numbers $\left[\widetilde{U}, b^{\dagger} b\right]=\left[\widetilde{U}, a_{1}^{\dagger} a_{1}+a_{2}^{\dagger} a_{2}\right]$ $=0$, which is consistent with the absence of energy exchange.

This transformation properly accounts for the subdynamics of the apparatus modes. When there is no photon in mode $b$ (object out), $\widetilde{U}=\exp \left[\delta\left(a_{2}^{\dagger} a_{1}-a_{1}^{\dagger} a_{2}\right)\right]$, and there is normal interference in the series of Mach-Zehnder interferometers. When there is a photon in $b$ (object in), $\widetilde{U}$ is the identity. The interference is prevented by the frequent removal of the field in mode $a_{2}$ and its replacement by vacuum. In the limit $N \rightarrow \infty$, the reflectivity of each beam splitter tends to one and the field in mode $a_{1}$ does not change.

Now, we can examine the transformation of object observables. The output complex amplitude operator for the field mode $b$ is

$$
\widetilde{U}^{\dagger} b \widetilde{U}=e^{i A B} b
$$

where

$$
\begin{gathered}
A=i\left(a_{2}^{\dagger} a_{1}-a_{1}^{\dagger} a_{2}\right), \\
B=\frac{\delta}{2}\left[\cos \left(\pi b^{\dagger} b\right)+\sqrt{2} \sin \left(\frac{\pi}{2} b^{\dagger} b+\frac{\pi}{4}\right)\right] .
\end{gathered}
$$

This transformation is a phase shift that depends on the field state in modes $a_{1}$ and $a_{2}$. Leaving aside the case $|0,0\rangle_{a}, A$ always presents fluctuations in the states $|\psi, 0\rangle_{a}$. These quantum fluctuations lead to a random phase shift in Eq. (3.3), so the object phase is disturbed by the observation. This is consistent, since the photon number is not modified and the variable suffering the back action should be its complementary.

The phase of the field mode $b$ is in fact the relative phase of the coherent superposition of the object-in and object-out situations. This means that the fluctuating phase shift (3.3) represents a decoherence effect so that the initial pure state is replaced by the density matrix

$$
|\alpha|^{2}|0\rangle\left\langle\left. 0|+| \beta\right|^{2} \mid 1\right\rangle\langle 1|+\cos \delta\left(\alpha^{*} \beta|1\rangle\left\langle 0\left|+\alpha \beta^{*}\right| 0\right\rangle\langle 1|\right) .
$$

However, this decoherence induced by the object-apparatus interaction is not irreversible. Whenever $\cos \delta=1$ the object will return to its initial state, recovering the coherence of the

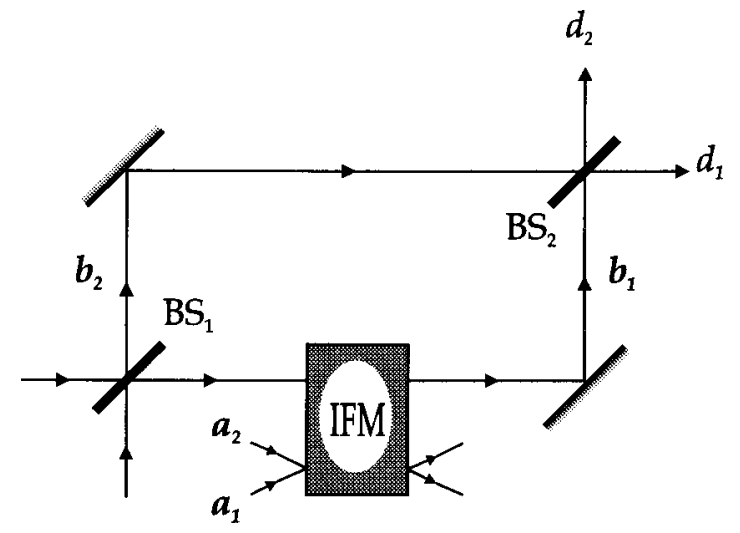

FIG. 2. Mach-Zehnder arrangement measuring the effect on the observed system (mode $b_{1}$ ) of the interaction-free detector (represented as IFM) in Fig. 1. The beam splitters $\mathrm{BS}_{1}$ and $\mathrm{BS}_{2}$ are $50 \%$. The interferometer is illuminated by a single input photon. The two internal paths are the field modes $b_{1}$ and $b_{2}$, while the outputs are the modes $d_{1}$ and $d_{2}$. The interaction-free measurement is applied to the internal mode $b_{1}$ acting in this way as a which-path detector.

quantum superposition. This is because of the small number of degrees of freedom of the apparatus [8].

For some particular object states the interaction has no consequences on the object state. These are mixed states with a diagonal density matrix in the number basis $\rho_{b}=p|1\rangle\langle 1|+(1-p)| 0\rangle\langle 0|$, with $1 \geqslant p \geqslant 0$. For these states we have $\left[\widetilde{U}, \rho_{b}\right]=0$ and they remain undisturbed. From the perspective followed here, this happens because these states have completely random phase and therefore the phase fluctuations caused by the observation leave them unchanged. These states represent the case of classical objects with probability $p$ of being present considered in previous works. These initial states will describe also the actual situation when dealing with macroscopic objects. This is because macroscopic objects cannot be left in a superposition state like the one considered here for more than negligible times at all scales.

\section{INTERFEROMETRIC OBSERVATION OF THE INDUCED PHASE SHIFT}

In the previous section we have shown that the effective interaction (3.2) will cause observable changes on the object state, provided it can be prepared in a coherent superposition. The transformation (3.3) is a phase shift of the field mode $b$ caused by its interaction with the interfering photon. This phase shift would be properly observed in an interferometric arrangement. To this end we will consider the Mach-Zehnder interferometer with $50 \%$ beam splitters schematized in Fig. 2. The two internal paths are the field modes $b_{1}$ and $b_{2}$ and the output ports are the field modes $d_{1}$ and $d_{2}$. The interaction-free detection previously analyzed can be applied to mode $b_{1}$.

First we assume that the interaction-free detector is absent. When the interferometer is illuminated by a single photon at one of the input ports, the field state in modes $b$ which is incident on $\mathrm{BS}_{2}$ is

$$
\frac{1}{\sqrt{2}}\left(|1,0\rangle_{b}+e^{i \phi}|0,1\rangle_{b}\right),
$$


where $\phi$ represents the phase difference between the two arms. The interference is observed as the dependence of the output intensities on $\phi$. Equivalently, we can calculate the output photon numbers to get $\left\langle d_{1}^{\dagger} d_{1}-d_{2}^{\dagger} d_{2}\right\rangle=\cos \phi$.

Now we consider that the interaction-free detection is applied to the mode $b_{1}$. This is a which-path detector, determining which of the two possible paths the photon follows through the interferometer. When $\epsilon=\pi /(2 N)$, the state (4.1) is replaced by

$$
\frac{1}{\sqrt{2}}\left(|1,0\rangle_{b}|1,0\rangle_{a}+e^{i \phi}|0,1\rangle_{b}|0,1\rangle_{a}\right) .
$$

The path is determined with certainty because the detection of the photon in modes $a_{1}$ or $a_{2}$ reveals that the path followed was $b_{1}$ or $b_{2}$, respectively. However, the interference is lost because the reduced state in modes $b$ no longer depends on $\phi$. This can be explained in terms of the random phase change caused by the observation. Using Eqs. (3.1) and (3.2), the state (4.2) can be written as

$$
\begin{aligned}
& \frac{1}{\sqrt{2}}\left(|1,0\rangle_{b}+e^{i \phi} e^{-i(\pi / 2) A}|0,1\rangle_{b}\right)|1,0\rangle_{a} \\
& \quad=\sum_{\lambda= \pm 1} \frac{1}{2}\left(|1,0\rangle_{b}+e^{i \phi} e^{-i(\pi / 2) \lambda}|0,1\rangle_{b}\right)|A=\lambda\rangle_{a},
\end{aligned}
$$

where $|A= \pm 1\rangle_{a}=(1 / \sqrt{2})\left(|1,0\rangle_{a} \pm i|0,1\rangle_{a}\right)$ are the onephoton eigenstates of $A$. The photon-number difference at the output is

$$
\left\langle d_{1}^{\dagger} d_{1}-d_{2}^{\dagger} d_{2}\right\rangle=\frac{1}{2} \sum_{\lambda= \pm 1} \cos (\phi-\lambda \pi / 2)=0 .
$$

We can see that the output intensities result from the incoherent superposition of two contributions with relative phases $\phi+\pi / 2$ and $\phi-\pi / 2$ that cancel, leading to output intensities independent of $\phi[9]$.

These two contributions can be split and then it is possible to observe them independently, provided that $A$ is actually measured. This can be achieved by mixing $a_{1}$ and $a_{2}$ at a symmetrical $50 \%$ beam splitter and detecting the output photon numbers. Then, the outcomes of the photon-number measurements on the output modes $d_{1}$ and $d_{2}$ can be classified according to the result of the measurement of $A$, leading to two conditional interference patterns

$$
\left.\left\langle d_{1}^{\dagger} d_{1}-d_{2}^{\dagger} d_{2}\right\rangle\right|_{A=\lambda}=\frac{1}{2} \cos (\phi-\lambda \pi / 2),
$$

which are shifted by $\pm \pi / 2$ with respect to the interference pattern provided by the unobserved state (4.1). This procedure is known as the erasure of the information because the result of the measurement of $A$ does not provide any information about the path followed by the photon within the interferometer [10].

\section{CONCLUSIONS}

The preceding example illustrates the trace left in the observed system by an interaction-free measurement. This is an evidence of the interaction between the object and the apparatus during the detection process. We have shown that it alters the relative phase of a coherent superposition of the object states discriminated by the detection arrangement. This results in a decoherence effect changing an initial coherent superposition into the corresponding statistical mixture. However, this decoherence induced by the interaction is not irreversible due to the small dimension of the apparatus.

All this means that the effective interaction can only be unveiled when the object is treated quantum mechanically and provided that it can be initially prepared in a coherent superposition. This is essential since the object variable disturbed cannot appear in a classical or in a corpuscular description of the process.

The interaction-free example considered in this work is an idealized scheme mainly due to the high value assumed for the Kerr coupling. Nevertheless we think that it can be useful to illustrate the main features of the process. The conclusions obtained here should remain valid when considering more realistic realizations.

\section{ACKNOWLEDGMENT}

We wish to thank Professor G. García-Alcaine for a careful reading of the manuscript and helpful discussions.
[1] A. C. Elitzur and L. Vaidman, Found. Phys. 23, 987 (1993); L. Vaidman, Quantum Opt. 6, 119 (1994).

[2] P. Kwiat, H. Weinfurter, T. Herzog, and A. Zeilinger, Phys. Rev. Lett. 74, 4763 (1995); P. Kwiat, H. Weinfurter, T. Herzog, A. Zeilinger, and M. Kasevich, Ann. (N.Y.) Acad. Sci. 755, 383 (1995).

[3] R. H. Dicke, Am. J. Phys. 49, 925 (1981); L. Hardy, Phys. Lett. A 167, 11 (1992).

[4] M. Pavičić, Phys. Lett. A 223, 241 (1996).

[5] F. X. Kärtner and H. A. Haus, Phys. Rev. A 47, 4585 (1993).
[6] H. Paul and M. Pavičić, Int. J. Theor. Phys. 35, 2085 (1996); J. Opt. Soc. Am. B 14, 1275 (1997).

[7] B. Yurke, S. L. McCall, and J. R. Klauder, Phys. Rev. A 33, 4033 (1986).

[8] J. M. Raimond, M. Brune, and S. Haroche, Phys. Rev. Lett. 79, 1964 (1997).

[9] S. M. Tan and D. F. Walls, Phys. Rev. A 47, 4663 (1993).

[10] M. O. Scully, B.-G. Englert, and H. Walther, Nature (London) 351, 111 (1991). 